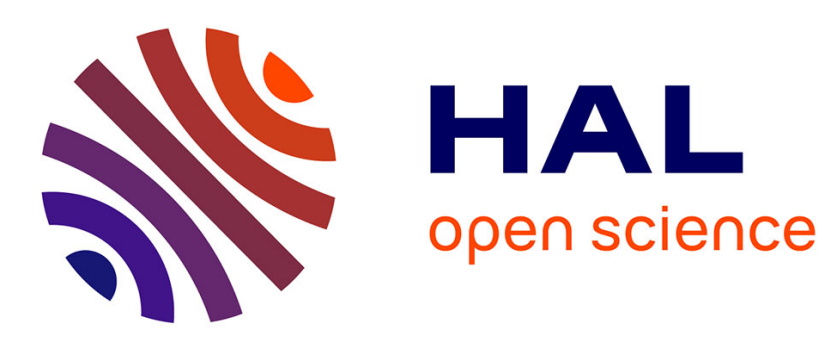

\title{
Micromechanical modelling and experimental investigation of random discontinuous glass fiber polymer matrix composites
}

A. Bouaziz, F. Zaïri, M. Naït-Abdelaziz, J.M. Gloaguen, J.M. Lefebvre

\section{To cite this version:}

A. Bouaziz, F. Zaïri, M. Naït-Abdelaziz, J.M. Gloaguen, J.M. Lefebvre. Micromechanical modelling and experimental investigation of random discontinuous glass fiber polymer matrix composites. Composites Science and Technology, 2007, 67 (15-16), pp.3278. 10.1016/j.compscitech.2007.03.031 . hal00498984

\section{HAL Id: hal-00498984 \\ https://hal.science/hal-00498984}

Submitted on 9 Jul 2010

HAL is a multi-disciplinary open access archive for the deposit and dissemination of scientific research documents, whether they are published or not. The documents may come from teaching and research institutions in France or abroad, or from public or private research centers.
L'archive ouverte pluridisciplinaire HAL, est destinée au dépôt et à la diffusion de documents scientifiques de niveau recherche, publiés ou non, émanant des établissements d'enseignement et de recherche français ou étrangers, des laboratoires publics ou privés. 


\section{Accepted Manuscript}

Micromechanical modelling and experimental investigation of random discontinuous glass fiber polymer matrix composites

A. Bouaziz, F. Zaïri, M. Naït-Abdelaziz, J.M. Gloaguen, J.M. Lefebvre

PII: S0266-3538(07)00141-8

DOI: 10.1016/j.compscitech.2007.03.031

Reference:

CSTE 3652

To appear in:

Composites Science and Technology

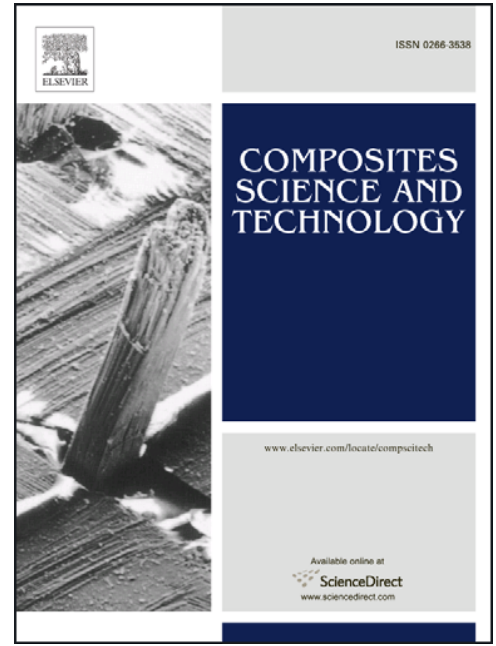

Received Date: 13 October 2006

Revised Date: $\quad 28$ February 2007

Accepted Date: $\quad 28$ March 2007

Please cite this article as: Bouaziz, A., Zaïri, F., Naït-Abdelaziz, M., Gloaguen, J.M., Lefebvre, J.M., Micromechanical modelling and experimental investigation of random discontinuous glass fiber polymer matrix composites, Composites Science and Technology (2007), doi: 10.1016/j.compscitech.2007.03.031

This is a PDF file of an unedited manuscript that has been accepted for publication. As a service to our customers we are providing this early version of the manuscript. The manuscript will undergo copyediting, typesetting, and review of the resulting proof before it is published in its final form. Please note that during the production process errors may be discovered which could affect the content, and all legal disclaimers that apply to the journal pertain. 


\title{
Micromechanical modelling and experimental investigation of random discontinuous glass fiber polymer matrix composites
}

\section{A. Bouaziz ${ }^{1}$, F. Zaïri ${ }^{*}$, M. Naït-Abdelaziz ${ }^{1}$, J.M. Gloaguen², J.M. Lefebvre ${ }^{2}$}

${ }^{1}$ Laboratoire de Mécanique de Lille (UMR CNRS 8107), USTL, Polytech'Lille, Avenue P. Langevin, 59655 Villeneuve d'Ascq Cedex, France

${ }^{2}$ Laboratoire de Structure et Propriétés de l'Etat Solide (UMR CNRS 8008), USTL, Bâtiment

C6, 59655 Villeneuve d'Ascq Cedex, France

* Corresponding author.

E-mail address: fahmi.zairi@ polytech-lille.fr

Phone number: (33)328767460

Fax number: (33)328767301

\begin{abstract}
Videomeasurements were used to estimate the damage in chopped random glass fiber polymer matrix composites. In order to predict the overall mechanical behaviour, voiding evolution induced by fiber debonding is incorporated into a micromechanics-based constitutive model. The comparison between the experimental data and the numerical predictions shows a very good agreement.
\end{abstract}

Keywords: A. Polymer-matrix composites (PMCs); B. Modelling; B. Microstructure; B. Debonding; D. Videoextensometry

\section{Introduction}

Fiber-reinforced polymer matrix composites are widely used in industry as structural materials and then there is an evident need for the prediction of their mechanical properties. In this kind of materials, various damage phenomena can occur such as matrix cracking, interfacial debonding, fiber pull-out and fiber fracture. The predominant damage mechanism 
may vary according to fiber volume fraction and aspect ratio, orientation and distribution in the composite, strengths of the interface and each constituent, and loading mode. Progressive damage accumulation in the composite is known to affect the overall mechanical properties. In order to estimate the overall response of the material, the accumulated damage must therefore be included in the constitutive relations. Furthermore, in order to achieve a rigorous description of material, the constitutive equations must be derived from micromechanical considerations. However, due to the complexity of the microstructure, the damage mechanisms in fiber-reinforced polymer matrix composites are not fully understood from the experimental point of view. Nevertheless, some micromechanical analyses have been proposed in literature to simulate the damage response of discontinuously fiber-reinforced composites. Meraghni and Benzeggagh [1] and Meraghni et al. [2-3] identified damage mechanisms in a random discontinuous fiber-reinforced composite based on acoustic emission. The authors proposed a micromechanical analysis based on a modified version of the Mori-Tanaka model to investigate the effect of damage mechanisms on the overall response. Similar analyses were made by Fitoussi et al. [4-5] and Derrien et al. [6] relying on the ultrasonic method and the Mori-Tanaka approach. Considering fibers randomly oriented in a ductile polymer matrix, Lee and Simunovic [7-9] recently proposed a micromechanical model. In all these investigations, the damage evolution was introduced into the micromechanical approach from probabilistic considerations in the form of a Weibull statistical density function. This probability function introduces additional parameters generally calibrated on an experimental stress-strain curve.

In the present paper, the damage behaviour of glass mat fiber-reinforced polymer matrix composite is investigated using a combined approach of micromechanical modelling and experimental characterization. A representative composite, consisting of a polyester matrix incorporating various volume fractions of randomly oriented glass fibers, was chosen. Rather 
than using the methods (acoustic emission and ultrasonic) usually employed, an alternative technique based on volume strain variation is retained to quantify the progressive interfacial damage in the material. The progressive damage observed on the macroscopic level was identified at the microscopic level by SEM observations. Damage evolution is introduced into a micromechanical model based on the Eshelby formulation and the ensemble-volume averaging homogenization process. The resulting micromechanical model is implemented numerically and used to simulate uniaxial loading for the sake of comparison with experimental results.

\section{Experiments}

\subsection{Material}

The composite under study consists of randomly oriented chopped (E-glass) fibers embedded in an unsaturated polyester matrix. The material was fabricated by compression molding at room temperature from mat densities of 300 and $450 \mathrm{~g} / \mathrm{m}^{2}$. Each fiber is constituted by a bundle of 50 filaments of about $10 \mu \mathrm{m}$ in diameter and $50 \mathrm{~mm}$ in length. The fibers, randomly distributed in the plane of the material, exhibit an aspect ratio $\alpha$ of about 100. The fiber volume fraction, obtained by burning off the polyester matrix, is found to be 13 and $21 \%$. For convenience in the remaining of the paper, the glass fiber reinforced polyester (GFRP) composites are coded as follows: GFRP-21R and GFRP-13R for the 21 and $13 \%$ fiber volume fractions respectively.

\subsection{Experimental method}


In literature, the damage response in fiber reinforced polymer composites is experimentally addressed from acoustic emission or ultrasonic techniques. In these methods, the void nucleation at the interfaces is not explicitly quantified and is completely ignored in the associated modelling. In this paper, video-controlled tensile tests and scanning electron microscope (SEM) observations were achieved in order to understand the basic mechanisms governing deformation in the composite material.

Parallelepipedic plates were machined to the specimen shape presented in Fig. 1. Rather than using a normalized specimen with constant cross-section, we have designed this specific geometry of the specimen in order to localize the damage in the central part. The large radius of curvature allows to be freed from the stress triaxiality dependence.

The mechanical behaviour of the composite material was determined at room temperature by tensile tests on an Instron $^{\odot}$ (model 5800) machine equipped with videotraction ${ }^{\odot}$ extensometer. The videotraction ${ }^{\odot}$ set-up consists of a CCD videocamera and an unit for image digitizing and analyzing as schematically represented in Fig. 2 [10-11]. Contrary to standard strain measurement methods such as gauge which impose a measure with contact, the optical extensometer allows to avoid the potential damage initiation on the specimen surface. The technique consists in following, in real time, the location of the center of gravity of ink marks drawn on the sample surface (Fig. 1). Five round markers (A, B, C, D and E) are aligned along the tensile axis 1 , each located $1 \mathrm{~mm}$ apart (center-to-center). Two others (F and $\mathrm{G})$ are placed on the perpendicular axis 2 and are separated as much as possible. Their diameter is about of $0.5 \mathrm{~mm}$. The objective of the method is to determine the three principal strains in the representative volume element (RVE) defined by the three transversal markers [11]. The thickness of the RVE is about $0.2 \mathrm{~mm}$. The surface containing the seven markers is digitized and the coordinates of their gravity centers are provided 50 times per second with a precision of about 0.05 pixel, which gives a precision on the deformation of about 0.0002 . 
The axial strain is determined from the partial axial strains induced by the relative displacement of markers dispos ed along the axis 1 . The partial axial strains are given by:

$$
\begin{aligned}
& E_{11}(A B)=\frac{A B-A_{0} B_{0}}{A_{0} B_{0}} \\
& E_{11}(B C)=\frac{B C-B_{0} C_{0}}{B_{0} C_{0}} \\
& E_{11}(C D)=\frac{C D-C_{0} D_{0}}{C_{0} D_{0}} \\
& E_{11}(D E)=\frac{D E-D_{0} E_{0}}{D_{0} E_{0}}
\end{aligned}
$$

where $\left(A_{0} B_{0}, B_{0} C_{0}, C_{0} D_{0}, D_{0} E_{0}\right)$ and $(A B, B C, C D, D E)$ are initial and extended distances defined in Fig. 1. The axial strain in the RVE is obtained by a nonlinear polynomial interpolation from the four partial axial strains [11]:

$$
\mathrm{E}_{11}=\left[\text { nonlinear interpolation from } \mathrm{E}_{11}(A B), \mathrm{E}_{11}(B C), \mathrm{E}_{11}(C D), \mathrm{E}_{11}(D E)\right]_{(F C G)}
$$

The transversal strain is given by:

$$
E_{22}=E_{22}(F G)=\frac{F G-F_{0} G_{0}}{F_{0} G_{0}}
$$

where $F_{0} G_{0}$ and $F G$ are initial and extended distances of the transversal markers. Note that since only one camera is used, $E_{22}$ and $E_{33}$ are assumed equal.

Once the three principal strains are measured in the RVE, the local volume variation measuring the dilatation in the material can be calculated from the following relation:

$$
E_{v}=\frac{V-V_{0}}{V_{0}}=\left(1+E_{11}\right)\left(1+E_{22}\right)\left(1+E_{33}\right)-1
$$

where $V_{0}$ and $V$ are the initial and current volume of the RVE.

The damaged local volume variation is given by:

$$
E_{v}^{d}=E_{v}-E_{v}^{e}
$$

where $E_{v}^{e}$ is the elastic part of the volume variation defined by: 


$$
E_{v}^{e}=(1-2 v) \frac{\Sigma_{11}}{E}
$$

In (6) $v$ is the Poisson's ratio, $E$ is the Young's modulus and $\Sigma_{11}$ is the stress given by:

$$
\Sigma_{11}=\frac{F}{S_{0}\left(1+E_{22}\right)\left(1+E_{33}\right)}
$$

where $F$ is the applied load on the specimen and $S_{0}$ is the initial cross-section.

\subsection{Experimental results}

The local axial strain rate, during a tensile test at a constant cross-head speed of $0.2 \mathrm{~mm} / \mathrm{min}$, is displayed in Fig. 3a as a function of time. The global strain rate is determined from the cross-head speed and the initial grip to grip distance $(80 \mathrm{~mm})$. It is clear that the local strain rate is not constant during deformation. Indeed, it shows a decrease followed by an increase due to an heterogeneous deformation. In order to model the local behaviour of the damaged composites for a strain driven test, it is fundamental to keep the local strain rate constant. The system used in this study is able to control the local strain rate. Fig. $3 b$ shows result of a test at

a local constant strain rate of $4.10^{-5} \mathrm{~s}^{-1}$. Monitoring of the cross-head speed by the videotraction system is clearly seen. This control is achieved in real time with the simultaneous data measurements.

The stress-strain curves of composites conducted at a local constant strain rate of $10^{-4} \mathrm{~s}^{-1}$ are given in Fig. 4. The strain at break of these composite specimens is of the order of 0.01 . Therefore, these chopped random fiber composites are brittle in nature.

As shown in Fig. 5, a whitening phenomenon is observed in the central part of the specimen. This phenomenon is generally associated in the literature with voids occuring in the material. The whitening appears because the void size is larger than the light wavelength (about 
$0.6 \mu \mathrm{m})$. It provides an optical verification of nucleation and propagation of voids in the material.

The damaged volume variation is presented as a function of axial strain in Fig. 4. The nucleation and growth stages of microvoiding are both revealed in this figure. It is evident that there exists an incubation strain where no damaged volume strain is observed. Note that for GFRP-21R, there is a small increase and decrease $\delta$ the damaged volume strain at the beginning of the loading; this measure has however no meaning since it is in the scattering range of the measurement system. The incubation strain depends on fiber volume fraction since it is equal to 0.004 for GFRP-21R and 0.0055 for GFRP-13R. In this incubation period, the volume variation is associated to the purely elastic dilatation due to the Poisson effect. Subsequently, an exponential damage increase is observed and the behaviour is approximately the same for the two materials. The scaling of the volume strain depends on fiber volume fraction. For an axial strain of 0.01, a damaged volume variation of 0.007 for GFRP -21R and 0.005 for GFRP-13R is found.

In order to identify the local damage mechanisms and the location of voids, microscopic observations on fracture surfaces at the RVE were achieved by SEM. Fig. 6 shows that the material contains numerous voids. These voids are localised around the fibers. The origin of these voids is attributed to the interfacial damage which seems to be the predominant microscopic damage mechanism in the composite. However, fiber fracture was also seen. Due to high hydrostatic stress, the region of broken fibers is also the site of void nucleation in the matrix. Furthermore, no significant matrix cracking was observed.

\section{Modelling}

\subsection{Constitutive relationships}


Traditional continuum mechanics cannot directly predict the correct behaviour of the composite since such an approach is based on continuity and homogeneity of the material. Indeed the concept of micromechanics is required to perform a rigorous analysis of the material and to predict the effect of microstructure on its overall mechanical properties. The constitutive law of the homogeneous medium equivalent to the heterogeneous material is established from a micromechanical modelling, taking into account both the existence of discontinuous randomly dispersed and oriented glass fibers, and damage evolution.

Chopped glass fibers are assumed to be elastic spheroids (prolate: $\alpha>1$ ) randomly dispersed and oriented in the elastic polymer matrix. As the spheroidal fibers are in the 1-2 plane, the composite as a whole is transversely isotropic.

The constitutive equations of the equivalent homogeneous medium is defined by:

$$
\mathbf{S}=\mathbf{C}: \mathbf{E}
$$

where $\mathbf{S}$ and $\mathbf{E}$ are the macroscopic stress and macroscopic strain tensors, respectively, and $\mathbf{C}$ is the fourth-order elastic stiffness tensor of the composite polymer (“:” signifies the tensor contraction).

The volume-averaged stress $\mathbf{S}$ and strain $\mathbf{E}$ tensors are given respectively by:

$$
\mathbf{S}=\frac{1}{V} \int_{V} \mathbf{s}(\mathbf{x}) d V \text { and } \mathbf{E}=\frac{1}{V} \int_{V} \mathbf{e}(\mathbf{x}) d V
$$

where $\mathbf{s}$ and $\mathbf{e}$ are the microscopic stress and microscopic strain tensors, respectively and $V$ is the volume of a RVE.

The fiber-reinforced composite presents initially perfect interfacial bonding between the matrix (phase 0) and fibers (phase 1). After its nucleation, damage (phase 2) is taken into account as an added phase in the composite. Therefore, the initial two phase composite becomes a three-phase composite from the onset of damage nucleation. 
According to the explicit formulation derived by $\mathrm{Ju}$ and Chen [12] for unidirectionally aligned spheroid-reinforced composites, the macroscopic stiffness tensor of the equivalent homogeneous medium is defined by the following expression:

$$
\mathbf{C}=\mathbf{C}_{0}\left[\mathbf{I}+\sum_{r=1}^{2} \mathbf{B}_{r}\left(\mathbf{I}-\mathbf{S}_{r} \mathbf{B}_{r}\right)^{-1}\right]
$$

where $\mathbf{I}$ is the fourth-rank identity tensor, $\mathbf{C}_{0}$ is the elasticity tensor of the matrix material, $\mathbf{S}_{r}$ is the Eshelby's tensor for phase $\mathrm{r}$ and $\mathbf{B}_{r}$ is a fourth-rank tensor defined by:

$$
\mathbf{B}_{r}=\phi_{r}\left[\mathbf{S}_{r}+\left(\mathbf{C}_{r}-\mathbf{C}_{0}\right)^{-1} \mathbf{C}_{0}\right]^{-1}
$$

where $\mathbf{C}_{r}$ and $\phi_{r}$ are respectively the elasticity tensor and the volume fraction of the phase $\mathrm{r}$.

The Eshelby's tensor $\mathbf{S}_{r}$ is a function of the Poisson's ratio of the matrix and the aspect ratio of the phase $r$. Its formulation for a spheroidal inclusion embedded in an isotropic linear elastic medium can be found in literature $[8,13]$.

Since for the studied material the fibers are randomly oriented in the 1-2 plane, it is necessary to introduce global axes denoted by the unprimed axes and local axes of each fibers denoted by the primed axes (Fig. 7). The random orientation of glass fibers can be then described by introducing the angle $\theta$ between the primed and unprimed axes:

$$
x_{i}=Q_{i j} x_{j}^{\prime}
$$

where the transformation matrix $Q_{i j}$ is given by:

$$
Q_{i j}=\left[\begin{array}{ccc}
\cos \theta & \sin \theta & 0 \\
-\sin \theta & \cos \theta & 0 \\
0 & 0 & 1
\end{array}\right]
$$

In (13), $\theta(0 \leq \theta \leq \pi)$ is the angle between $x_{1}$ and $x_{1}^{\prime}$. 
According to the studies of Tandon and Weng [14] and Lee and Simunovic [7], the orientational averaging process for all orientations for the macroscopic stiffness tensor can be written as:

$$
\langle\mathbf{C}\rangle=\int_{0}^{\pi} Q_{m i} Q_{n j} C_{m n p q} Q_{p k} Q_{q l} p(\theta) \sin \theta d \theta
$$

where $p(\theta)$ is the probability density function of the random orientation which is equal to $1 / \pi$ for uniformly random orientation.

In order to achieve more realistic behaviour predictions, the damage experimentally identified must be included in the modelling. Based on experimental evidence, the debonding along the interface between the matrix and fibers is the major failure mechanism in this material. The interfacial debonding under increasing deformation leads to the creation of new surfaces inducing void volume variation in the material. The increase of the void volume fraction is assumed to be a coupled effect of nucleation of voids and growth of these voids. The rate of void volume fraction is governed by the following evolution relation:

$$
\dot{\phi}_{2}=\dot{\phi}_{\text {nucl }}+\dot{\phi}_{\text {grow }}
$$

where $\dot{\phi}_{\text {nucl }}$ is the nucleation rate of voids and $\dot{\phi}_{\text {grow }}$ is the growth rate of existing voids.

The nucleation rate, linked to the local hydrostatic stress applied on the fiber, is expressed in a simple empirical way. The progressive partial debonding between fibers and the matrix can be taken into account with the Weibull statistical function [15]:

$$
P_{d}\left(\sigma_{1 h}\right)=1-\exp \left[-\left(\frac{\sigma_{1 h}}{\sigma_{u}}\right)^{m}\right]
$$

where $\sigma_{1 h}$ is the average internal hydrostatic stress of the fibers, $m$ and $\sigma_{u}$ are the Weibull parameters to be estimated from experiments. $m$ controls the shape of the Weibull function and $\sigma_{u}$ its scale. 
From the Eshelby inclusion theory, the stress inside fibers is assumed uniform and is given by [7]:

$$
\mathbf{S}_{1}=\mathbf{C}_{1}\left(\mathbf{I}-\mathbf{S}_{1} \mathbf{B}_{1} \phi_{1}^{-1}\right)\left(\mathbf{I}-\sum_{r=1}^{2} \mathbf{S}_{r} \mathbf{B}_{r}\right)^{-1}: \mathbf{E}
$$

The cumulative volume fraction of debonded fibers is then given by:

$$
\phi_{d}=\phi P_{d}\left(\sigma_{1 h}\right)
$$

where $\phi$ is the original volume fraction of bonded fibers.

After partial debonding, the debonded portion of the fiber does not transmit any more stresses to the matrix. The debonding process is mechanically complex because it introduces a local induced anisotropy which is not easy to account for. In order to simplify the problem, the debonded part of the fiber is substituted by an equivalent volume of matrix material. By this way, replacing elastic mechanical properties of the debonded fibers by that of the matrix leads to a global weakening of the composite. Moreover, the void volume fraction which is introduced as a third phase, also acts as supplementary weakening factor. The nucleation rate of interfacial voids by the debonding of fibers and the matrix is assumed to be controlled by the stress in the fibers:

$$
\dot{\phi}_{\text {nucl }}=\gamma \dot{\phi_{d}}=\gamma \phi \frac{\partial P_{d}\left(\sigma_{1 h}\right)}{\partial \sigma_{1 h}} \dot{\sigma}_{1 h}
$$

where $\gamma$ is a scaling coefficient for void nucleation rate and is assumed to be constant.

The growth rate of the previously nucleated microvoids at the interface is given by [16]:

$$
\dot{\phi}_{\text {grow }}=\phi_{\text {nucl }} \frac{3}{4 \mu} \dot{\Sigma}_{h}
$$

where $\mu$ is the shear modulus of the composite. Void growth is governed by the macroscopic hydrostatic stress. The voids are assumed to be spheroidal and to remain of uniform size during deformation. Furthermore, the interactions between the different phases are not taken into consideration in the present model. 
The model is introduced into a computer algorithm based on the strain driven scheme; the unknown macroscopic stress history is determined by a given macroscopic strain:

$$
\mathbf{S}_{t+\Delta t}=\mathbf{S}_{t}+\mathbf{C}: \Delta \mathbf{E}_{t+\Delta t}
$$

The incremental nonlinear equations for damage are solved using the Trapezoidal scheme:

$$
\phi_{2 t+\Delta t}=\phi_{2 t}+\Delta \phi_{2}
$$

where:

$$
\Delta \phi_{2}=\frac{\Delta t}{2}\left[\dot{\phi_{2 t}}+\dot{\phi}_{2 t+\Delta t}\right]
$$

The hydrostatic stress inside the fibers is computed according to (17) and the current volume fraction of bonded fibers is updated in the following way:

$$
\phi_{1 t+\Delta t}=\phi-\phi_{d t+\Delta t}
$$

The volume fractions of damage (22) and bonded fibers (24) are updated at each time increment step and are used to compute the progressive evolution of the stiffness tensor. After computation of the current stiffness tensor, the stresses are updated.

Considering the width $(23 \mathrm{~mm})$ of the specimens used in the mechanical tests (Fig. 1), the geometrical distribution of the fibers (nominal length of fibers is $50 \mathrm{~mm}$ giving $\alpha=100$ ) is expected to be inhomogeneous. Fig. 8 shows the influence of the aspect ratio $\alpha$ of the fibers on the predicted Young's modulus of the composite. It is clear that $\alpha$ influences strongly the Young's modulus for smaller values of $\alpha$ and its effect saturates when considering higher values (about $\alpha>30$ ). Therefore, it is worth assuming that all fibers have identical geometry.

\subsection{Comparison between predicted and measured behaviour}

The formulation given in the previous section is now applied to uniaxial loading condition.

The material properties of the unsaturated polyester matrix and fibers are: $E_{0}=4400 \mathrm{MPa}$, 
$v_{0}=0.35, E_{1}=72000 \mathrm{MPa}, v_{1}=0.25$. The Young's modulus and the Poisson's ratio of the matrix are measured from video-controlled tensile tests. The aspect ratio $\alpha$ of fibers is 100 . The parameters of the voids nucleation rate (19) are numerically determined using a least squares regression fitting on the accumulated damage curves observed in the experiments. They are found to be: $m=5, \sigma_{u}=118 \mathrm{MPa}$ and $\gamma=0.08$. These values are taken independent of the fiber volume fraction.

The simulated stress-strain responses and damaged volume strain are given in Fig. 9. The model predictions are in very good agreement with experiments. However, the final stage of damage, leading to brutal fracture, is not well represented. In this final stage, the simulated damage evolution underestimates the experimental data since no threshold stage is taken into account in the present modelling. Indeed, during the rupture various damage mechanisms (debonding, fiber pull-out and breakage) simultaneously accumulate leading to a sudden coalescence of voids which produce a drastic increase of the damaged volume strain.

The volume fraction evolution of debonded fibers predicted by the model is displayed in Fig. 10 for the two volume fractions of fibers. The case without damage representing an upper bound and the damaged case are shown in Fig. 11. The weakening effect due to damage can be clearly seen. This weakening is more emphasized for GFRP-21R than GFRP-13R.

\section{Conclusion}

The damage behaviour of chopped random glass fiber unsaturated polyester matrix composites was investigated. The experimental results have demonstrated the importance of taking into consideration the volume strain in the accurate characterization of composites damage, especially for the composites having a weak interfacial bond strength. The experimental results were used for the validation of a micromechanical model based on the 
ensemble -volume averaged homogenization procedure introduced by Lee and Simunovic [7].

The damage evolution law was incorporated into the modelling in terms of nucleation and growth of voids. The predictions of the micromechanical damage model were found in good agreement with the measured behaviour.

The modelling is clearly able to connect the complex microstructure and the dissipative mechanisms with the macroscopic behaviour of the material. Comparison with experiments was done only for in-plane mechanical behaviour. Therefore, data collection with other mechanical tests than uniaxial tension is required. Furthermore, the micromechanical constitutive model is going to be implemented into a finite ele ment code.

\section{References}

[1] Meraghni F, Benzeggagh ML. Micromechanical modeling of matrix degradation in randomly discontinuous-fibre composites. Comp Sci Tech 1995;55:171-186.

[2] Meraghni F, Blakeman CJ, Benzeggagh ML. Effect of interfacial decohesion on stiffness reduction in a random discontinuous-fibre composite containing matrix microcracks. Comp Sci Tech 1996;56:541-555.

[3] Meraghni F, Desrumaux F, Benzeggagh ML. Implementation of a constitutive micromechanical model for damage analysis in glass mat reinforced composite structures. Comp Sci Tech 2002;62:2087-2097.

[4] Fitoussi J, Bourgeois N, Guo G, Baptiste D. Prediction of the anisotropic damaged behavior of composite materials: introduction of multilocal failure criteria in a micro-macro relationship. Comp Mater Sci 1996;5:87-100.

[5] Fitoussi J, Guo G, Baptiste D. A statistical micromechanical model of anisotropic damage for SMC composites. Comp Sci Tech 1998;58:759-763.

[6] Derrien K, Fitoussi J, Guo G, Baptiste D. Prediction of the effective damage properties and failure properties of nonlinear anisotropic discontinuous reinforced composites. Comp Meth Appl Mech Eng 2000;185:93-107.

[7] Lee HK, Simunovic S. Modeling of progressive damage in aligned and randomly oriented discontinuous fiber polymer matrix composites. Comp PartB Eng 2000;31:77-86.

[8] Lee HK, Simunovic S. A damage constitutive model of progressive debonding in aligned discontinuous fiber composites. Int J Solids Struct 2001;38:875-895.

[9] Lee HK, Simunovic S. A damage mechanics model of crack-weakened, chopped fiber composites under impact loading. Comp PartB Eng 2002;33:25-34.

[10] François P, Gloaguen JM, Hue B, Lefebvre JM. Volume strain measurements by optical extensometry: application to the tensile behaviour of RT-PMMA. J Phys III 1994;4:321329. 
[11] G'Sell C, Hiver JM, Dahoun A. Experimental characterization of deformation damage in solid polymers under tension, and its interrelation with necking. Inter J Solids Struct 2002;39:3857-3872.

[12] Ju JW, Chen TM. Micromechanics and effective moduli of elastic composites containing randomly dispersed ellipsoidal inhomogeneities. Acta Mech 1994;103:103-121.

[13] Ju JW, Sun LZ. A novel formulation for the exterior-point Eshelby's tensor of an ellipsoidal inclusion. J Appl Mech 1999;66:570-574.

[14] Tandon GP, Weng GJ. Average stress in the matrix and effective moduli of randomly oriented composites. Comp Sci Tech 1986;27:111-132.

[15] Weibull W. A statistical distribution function of wide applicability. J Appl Mech 1951;18:293-297.

[16] Steenbrink AC, Van der Giessen E, Wu PD. Void growth in glassy polymers. J Mech Phys Solids 1997;45:405-437. 


\section{ACCEPTED MANUSCRIPT}

\section{Figure captions}

Fig. 1. Specimen dimensions (in $\mathrm{mm}$ ) and configuration of the seven markers for strains measurement.

Fig. 2. Schematic illustration of the optical extensometer system.

Fig. 3. Local and global strain rates for GFRP-13R: (a) non strain-controlled and (b) straincontrolled tests.

Fig. 4. Experimental stress-strain and damaged volume strain curves for: (a) GFRP-21R and (b) GFRP-13R.

Fig. 5. Photograph of a fractured GFRP-21R specimen.

Fig. 6. SEM photograph of the fractured surface for GFRP-21R.

Fig. 7. Local and global coordinates for a composite containing randomly located and oriented sphe roidal fibers.

Fig. 8. Influence of the shape parameter $\alpha$ on the predicted macroscopic Young's modulus.

Fig. 9. Predicted stress-strain and damaged volume strain curves compared to experimental data for: (a) GFRP-21R and (b) GFRP-13R.

Fig. 10. Predicted volume fraction of debonded fibers.

Fig. 11. Effect of damage on the overall behaviour. 


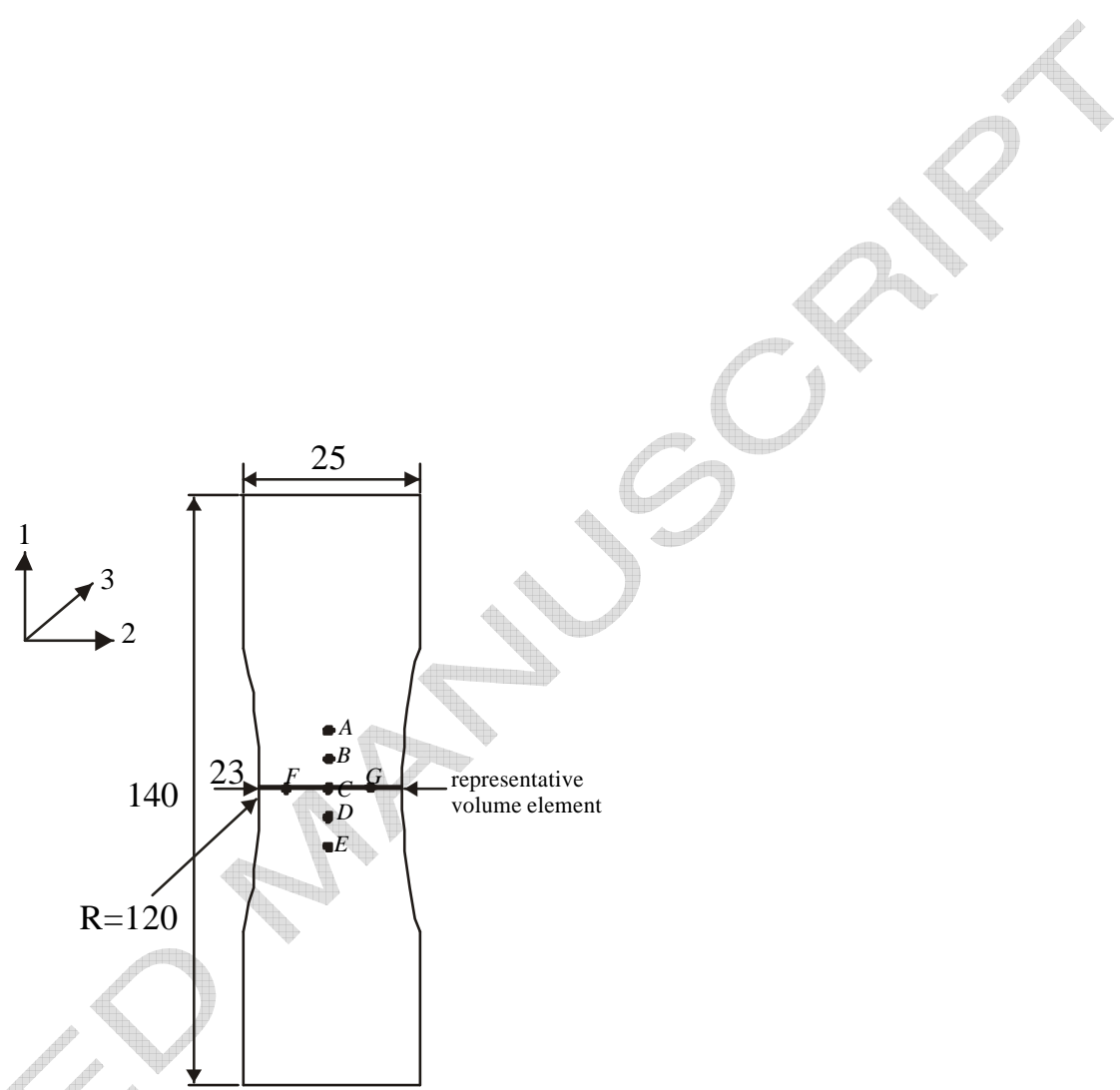

Fig. 1. Specimen dimensions (in $\mathrm{mm}$ ) and configuration of the seven markers for strains measurement. 


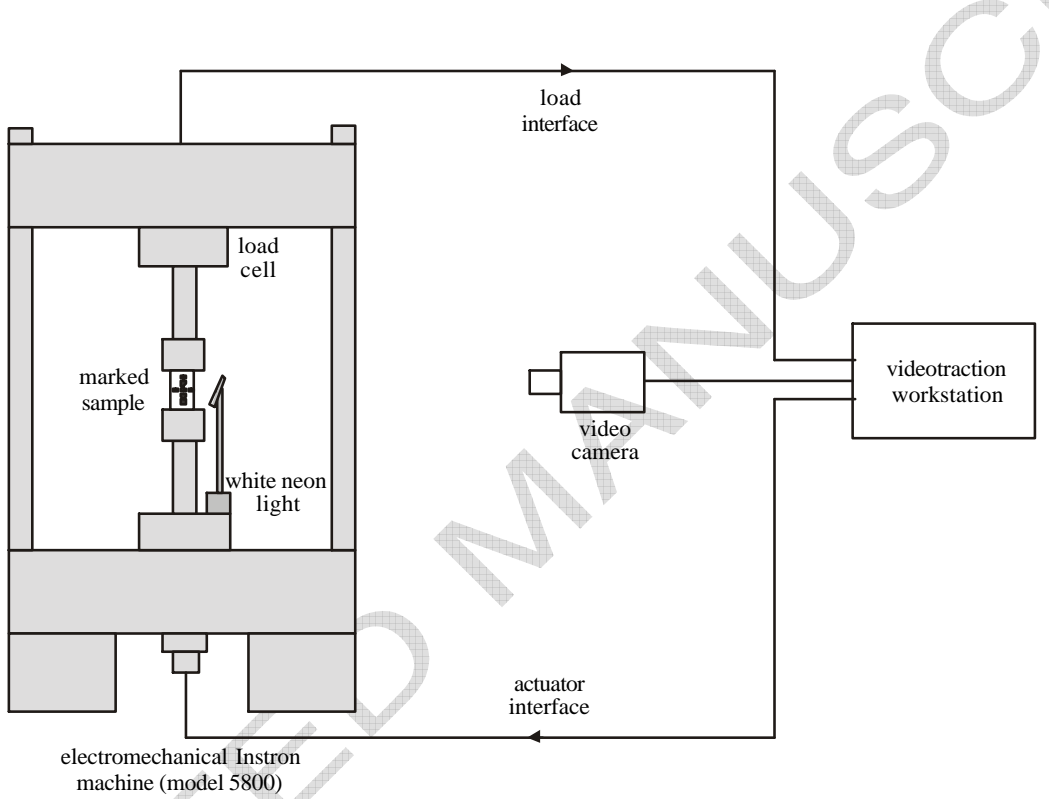

Fig. 2. Schematic illustration of the optical extensometer system. 


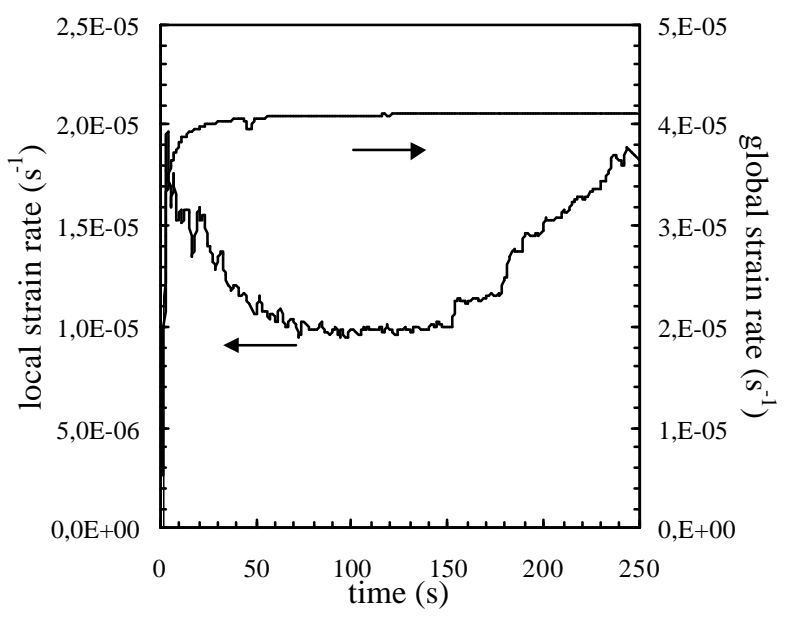

(a)

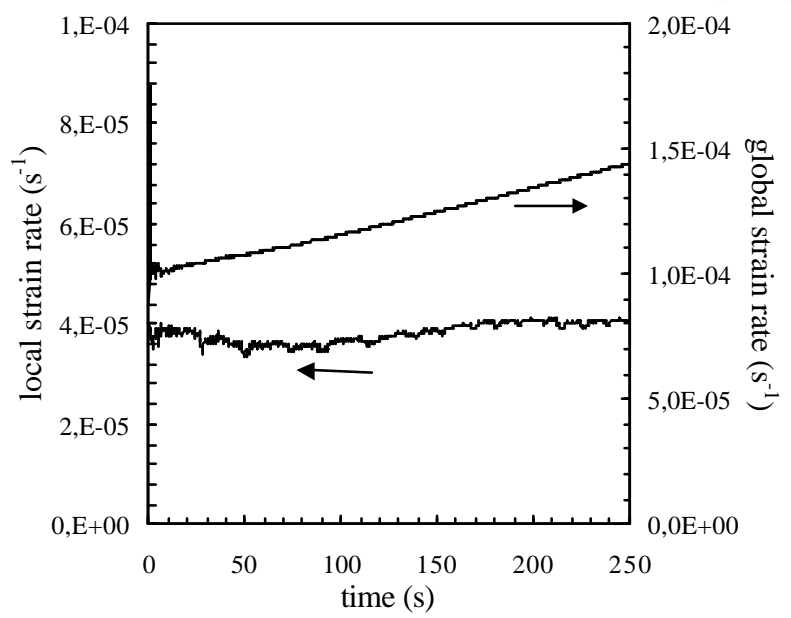

(b)

Fig. 3. Local and global strain rates for GFRP-13R:

(a) non strain-controlled and (b) strain-controlled tests. 


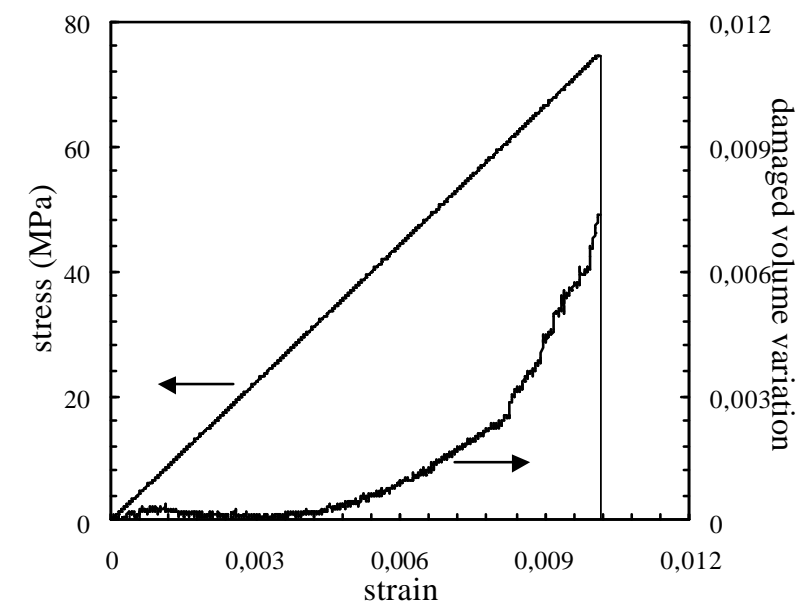

(a)

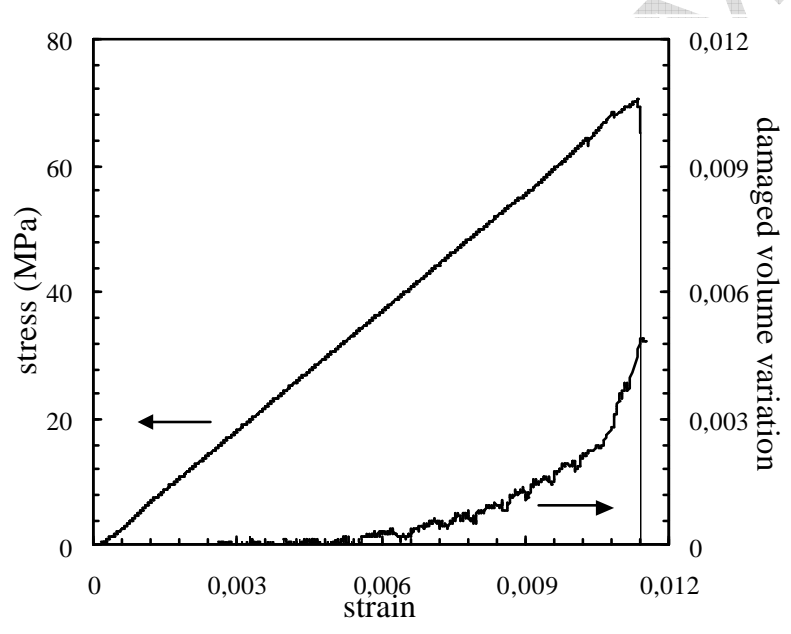

(b)

Fig. 4. Experimental stress -strain and damaged volume strain curves for: (a) GFRP-21R and (b) GFRP-13R. 


\section{ACCEPTED MANUSCRIPT}

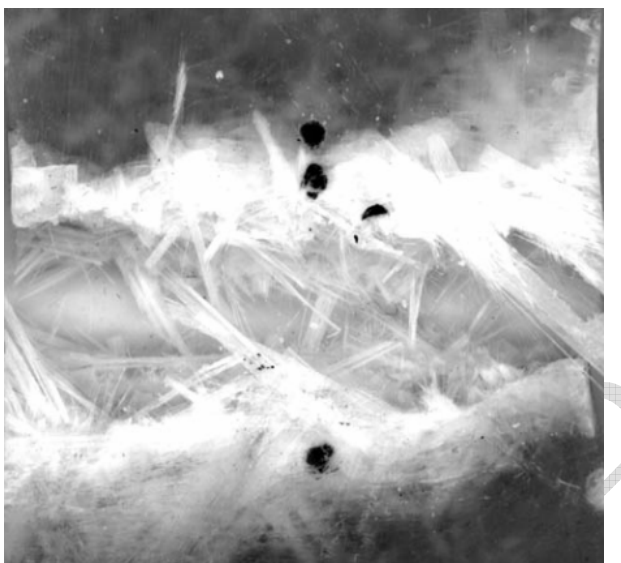

Fig. 5. Photograph of a fractured GFRP-21R specimen. 


\section{ACCEPTED MANUSCRIPT}

Fig. 6. SEM photograph of the fractured surface for GFRP-21R.
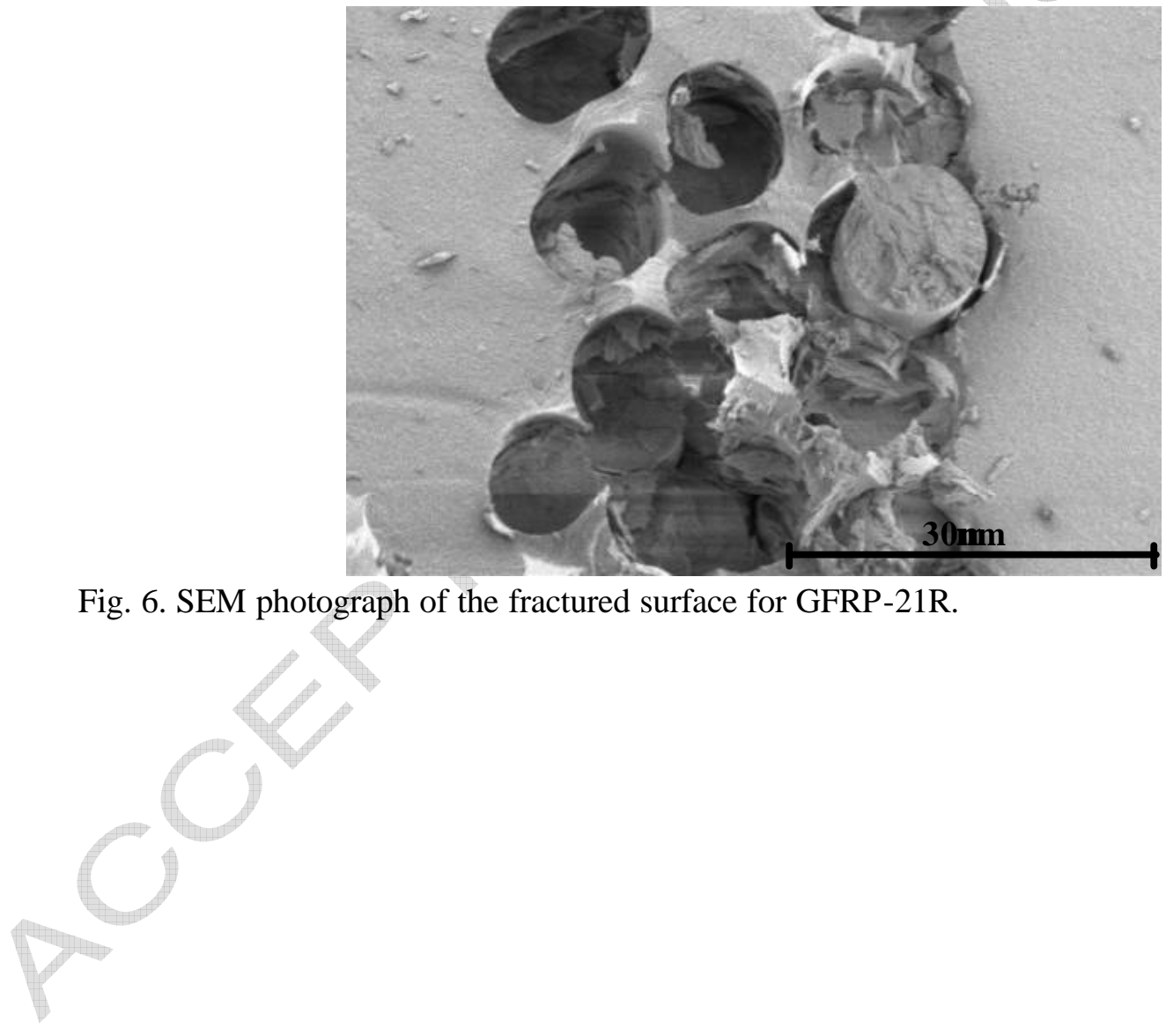


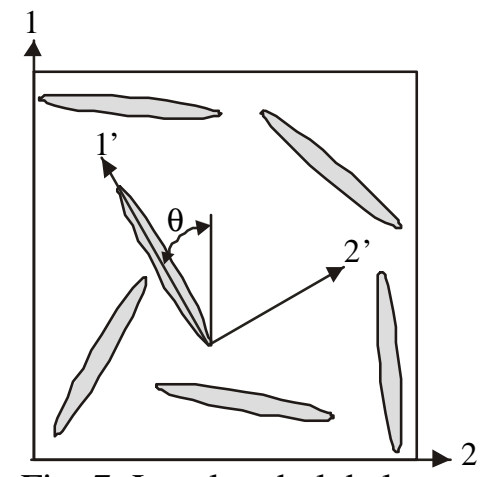

Fig. 7. Local and global coordinates for a composite containing randomly located and oriented spheroidal fibers. 


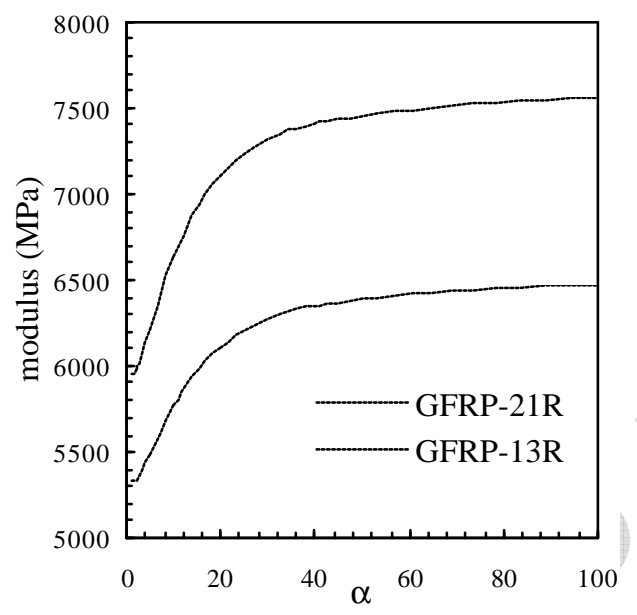

Fig. 8. Influence of the shape parameter $\alpha$ on the predicted macroscopic Young's modulus. 


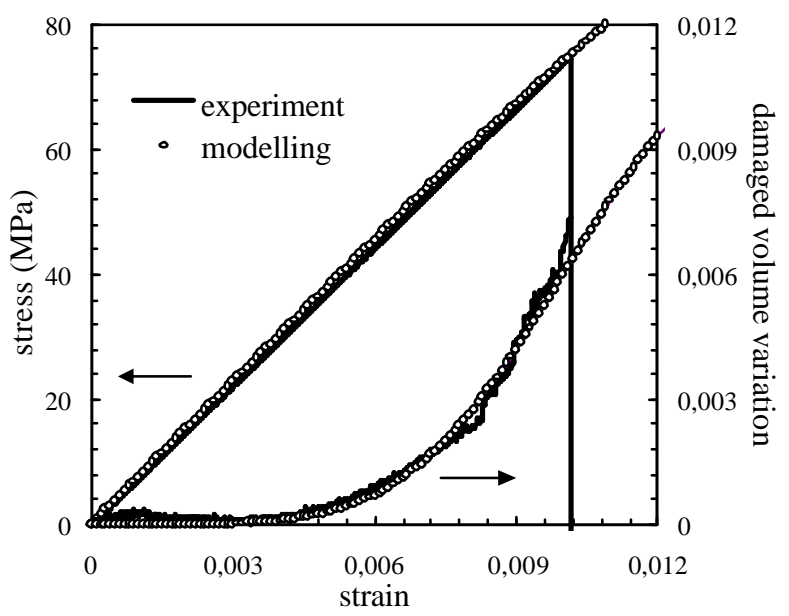

(a)

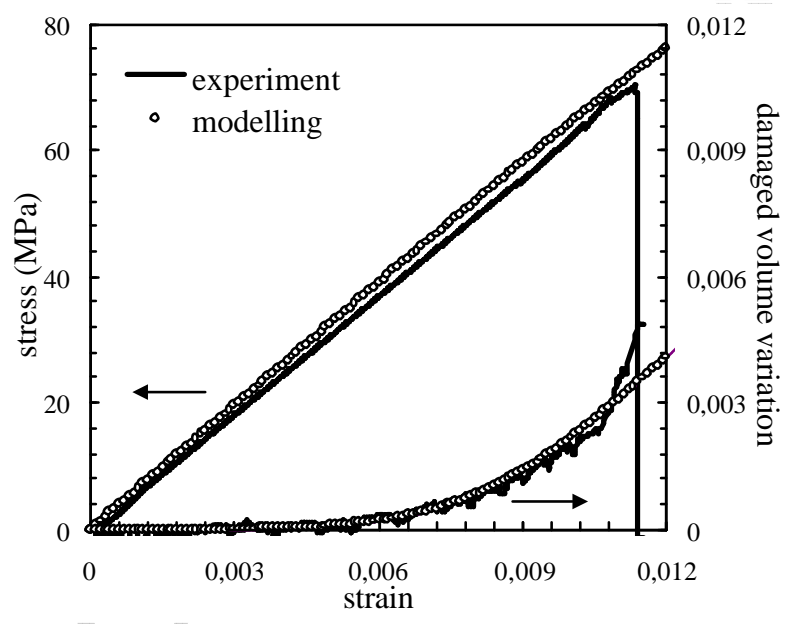

(b)

Fig. 9. Predicted stress-strain and damaged volume strain curves compared to experimental data for: (a) GFRP-21R and (b) GFRP-13R. 


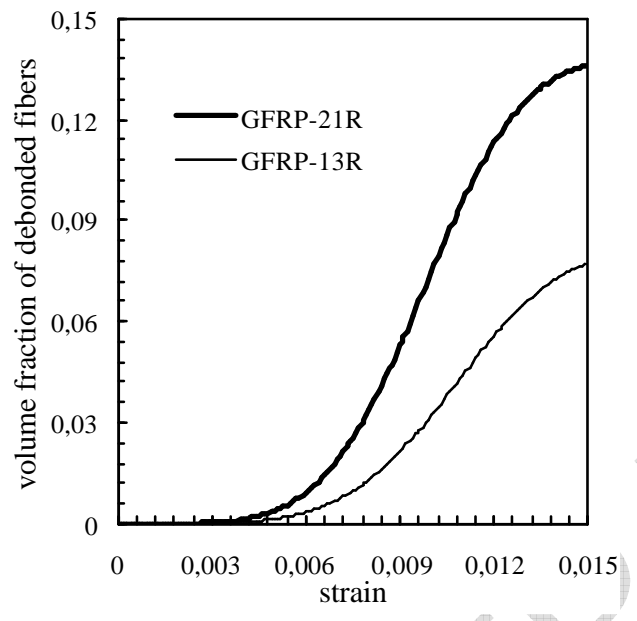

Fig. 10. Predicted volume fraction of debonded fibers. 


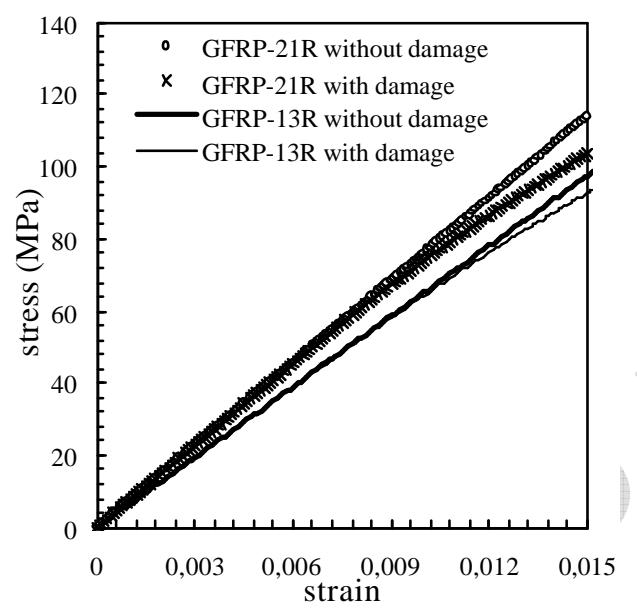

Fig. 11. Effect of damage on the overall behaviour. 